\title{
NATURAL TANNIN-BASED RIGID FOAMS AS INSULATION FOR DOORS AND WALL PANELS
}

\author{
G. Tondi ${ }^{1}$, A. Pizzi ${ }^{1}$, R. Olives ${ }^{2}$
}

\begin{abstract}
Tannin-based rigid foams were shown to be good thermal insulating materials for use in the cavity of hollow-core wooden doors and other wooden cavities. Their thermal insulation capacity is comparable to that of totally synthetic, oil-derived foams, such as polyurethanes, but with the advantage that they do not burn and thus do not emit toxic gases on burning. Exposure to the weather of wooden hollow core structures filled with tannin-based foams confirm that the wood in contact with the foam is not affected by the acidity of the foam due to the already previously demonstrated incorporation of the acid hardener used by coreaction with the hardened polymer network.
\end{abstract}

Keywords: tannins, foams, thermal insulation, wood, doors, construction

\section{INTRODUCTION}

Tannin based rigid foams are known for a long time as natural products resistant to fire like synthetic phenolic foams and for their application as floral foams (Meikleham and Pizzi1994, Tondi et al. 2008, Pizzi et al. 2008). These flavonoid-furanic foams are constituted of around 95\% of natural products. Furfuryl alcohol is obtained throughout catalytic reduction of furfural (Nagaraja et al. 2003) which is obtained by hydrolysis of the sugars from several agricultural crops (Aguilar et al. 2002), while tannin polyflavonoids are vegetal products obtained by water extraction from the wood and the bark of trees.

Condensed polyflavonoid tannins, due to their phenolic nature, can undergo some of the typical reactions of phenol such as reacting rapidly with formaldehyde under acid and alkaline conditions.

Condensed tannins are oligomers constituted by flavonoid repeating units as shown in Fig.1, mostly linked 4-6 or 4-8 to each other. The addition reaction of formaldehyde produce hydroxymethyl groups mainly at the free C8 or C6 sites (Pizzi 1994).

The most common commercial condensed tannins used for tannin foams are mimosa (Acacia mearnsii, or mollissima) bark tannin and quebracho (Schinopsis balansae and Schinopsi lorentzii) wood tannin. 


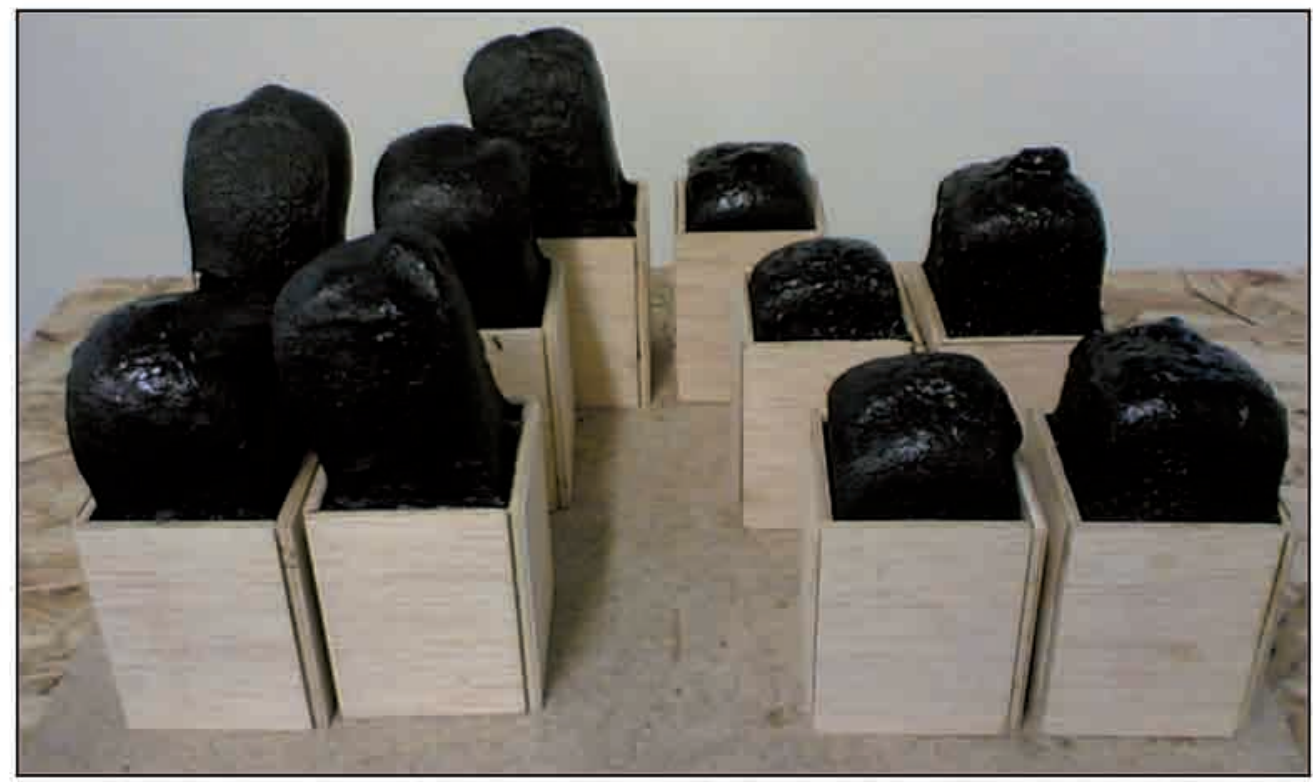

Figure 1. Appearance of plywood boxes within which tannin-based foams have been foamed and hardened at ambient temperature. Samples for exterior weather exposure.

The foaming process to obtain a tannin based rigid foam consists of three phases: Mixing, expansion and curing. Mixing is determinant to obtain an homogenous highly viscous bulk mix. All components (tannin extract, formaldehyde, furfuryl alcohol, blowing agent, additives and water) are added and mechanically stirred until homogeneity of system. The expansion phase starts when a catalyst (a strong acid) is added to the mix. The exothermic self-condensation of furfuryl alcohol and the condensation between furfuryl alcohol, tannin extract and formaldehyde take place simultaneously. At the same time the blowing agent, a low temperature boiling solvent, evaporates due to the temperature increase consequence of the exothermic reaction. This foams the whole mix which has already started to cure. The last step consists in the final curing of the tannin/furfuryl/formaldehyde network stabilising the foam in a few minutes.

This article deals with the application of these fire-resistant foams as a thermal insulating material within wood cavities, in particular hollow-core wooden doors.

\section{EXPERIMENTAL}

\section{Foam samples preparation and weather exposure in wood containers}

Three plywood boxes of $50 \times 50 \times 70 \mathrm{~mm}$ dimension and $4 \mathrm{~mm}$ thick, were filled with tannin-based rigid foam, with the liquid mix added and the foam allow to foam in situ. Tannin-based rigid foams have been prepared has follow:

Furfuryl alcohol (5,2g), formaldehyde $37 \%$ water solution $(3,7 g)$ and water $(3,0 \mathrm{~g})$ were mixed with $15 \mathrm{~g}$ of mimosa (Acacia mearnsii, or mollissima) tannin extract. When the agglomerate is homogeneous diethylether (1,5g) and toluene-4-sulphonic acid 65\% (6,0g) were added and sufficiently mixed for 10 second before the discharging into the 3 plywood boxes for foaming. The resin foams within 2 minutes of mixing with a temperature rise to about $40^{\circ} \mathrm{C}$ to yield a black foam of a density between 0,05 and 0,1 $\mathrm{g} \mathrm{cm}^{-3}$. After 10 minutes the skin of the foam was cut out and the remaining structure left for 4 hours to 
remove any residual blowing agent entrapped in the open-cell network. Then the box was closed with a plywood cover using a polyurethane glue for external application (Fig.1 and Fig.2).

The samples were exposed to natural weathering and analyzed after 1 day, 4 months and 8 months exposure.

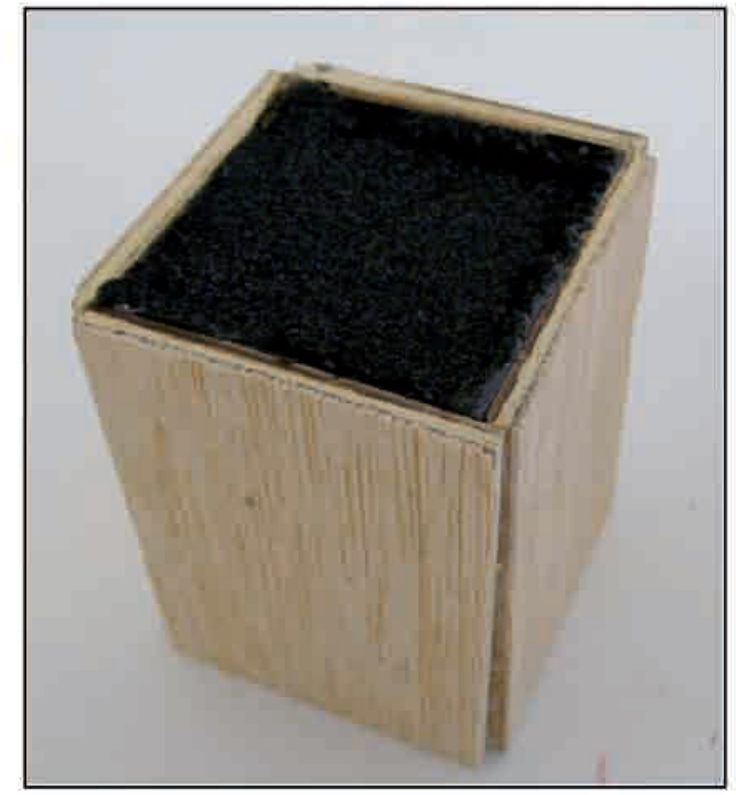

Figure 2. Appearance of plywood box immediately after the excess foam protruding from the box has been cut and just before the lid is applied.

\section{Foams thermal conductivity tests}

Samples of $25 \times 25 \times 25 \mathrm{~mm}$ dimensions of the tannin based foam were analysed for thermal conductivity.

The thermal conductivity measuring equipment, built exclusively for this type of test, consists of two square metal plates thermally stabilized by two heat exchangers-generators. The two metal plates were kept at the respective temperatures one of $60^{\circ} \mathrm{C}$ and the other of $10^{\circ} \mathrm{C}$. In between the two metal plates are situated two sample holders of $25 \times 25 \times 65 \mathrm{~mm}$. The sample holder on the hot metal plate is in macor (a ceramic material) while the sample holder placed on the cold metal plate is in stainless steel. The materials of the sample holders were chosen as it is better if the support materials present the nearest possible thermal conductivity to that of the samples to analyse.

The foam samples of $25 \times 25 \times 65 \mathrm{~mm}$ dimensions were placed within this structure. Eight thermocouples inserted at definite intervals in the sample holders and in the samples show the heat flow between the two metal plates through the samples. The position of the thermocouples are shown in Fig. 3. 


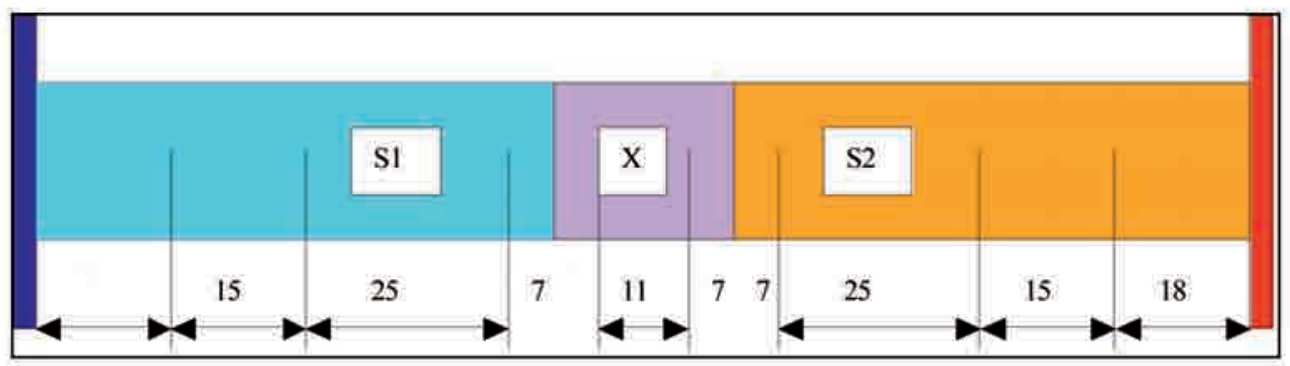

Figure 3. Schematic indication of the relative position of the insulating supports (S1 and S2) and sample $(X)$ in the equipment used for the determination of thermal conductivity. The number indicates in $\mathrm{mm}$ the relative positions of the temperature measuring probes.

The whole structure is protected by polystyrene foam blocks to avoid excessive side heat loss. The time to bring the equipment to stable equilibrium takes a minimum of two hours, depending from the initial state of the equipment and from the type of material to be tested. Once the system as reached steady state equilibrium one can obtain the value of the thermal conductivity $\lambda$ through a computer programme taking account all the necessary parameters. The samples were tested in two directions, namely the direction of foam growth and the direction normal to this latter. Several samples having different densities were analysed (Touloukian et al. 1970).

\section{Scanning Electron Microscopy}

Scanning electron microscopy was done using a Hitachi S-520 microscope at 30, 100 and 300 magnifications to characterize the foams.

Working distance of $8.2 \mathrm{~mm}$ and emission current of $10100 \mathrm{~nm}$ are the parameter used to collect the pictures of the tannin-based rigid foams.

\section{Formaldehyde emission}

Formaldehyde emission has been measured using the conditions reported in the Australian/New Zealand standard test for particleboards (AS/NZS 4266.16), adapted to foams. This test is well-known for reconstituted wood-based panels as "the desiccator method".

Nine pieces of around $450 \mathrm{~cm}^{2}$ of surface each have been left $24 \mathrm{~h}$ in a desiccator in the bottom of which have been stored $75 \mathrm{ml}$ of deionized water. Temperature of $20^{\circ} \mathrm{C}$ was maintained in a thermosetted room during the whole time of the experiment.

$25 \mathrm{ml}$ of the formaldehyde solution have been mixed with $25 \mathrm{ml}$ of Hantzsch reactive (prepared according to the AS/NZS 4266.16) in a $100 \mathrm{ml}$ flask and immersed in a $65^{\circ} \mathrm{C}$ water bath for 10 minutes.

The flask has been left for $1 \mathrm{~h}$ in a light protected environment before the absorbance of the solution is determined at a wavelength of $412 \mathrm{~nm}$. The value has been corrected using a water solution which has undergo the same procedure in a desiccator without foam samples.

\section{RESULTS AND DISCUSSION}

The appearance of tannin-based rigid foams observed by scanning electron microscopy is evident in Fig. 4. The foam has an open cell structure but restricted flow and access, the intercommunicating 
holes being rather small. These foams can be Taylor-made with a majority of closed cells or a even greater majority of open cells with different size interconnecting conducts (Meikleham and Pizzi 1994, Tondi et al. 2008, Pizzi et al. 2008).

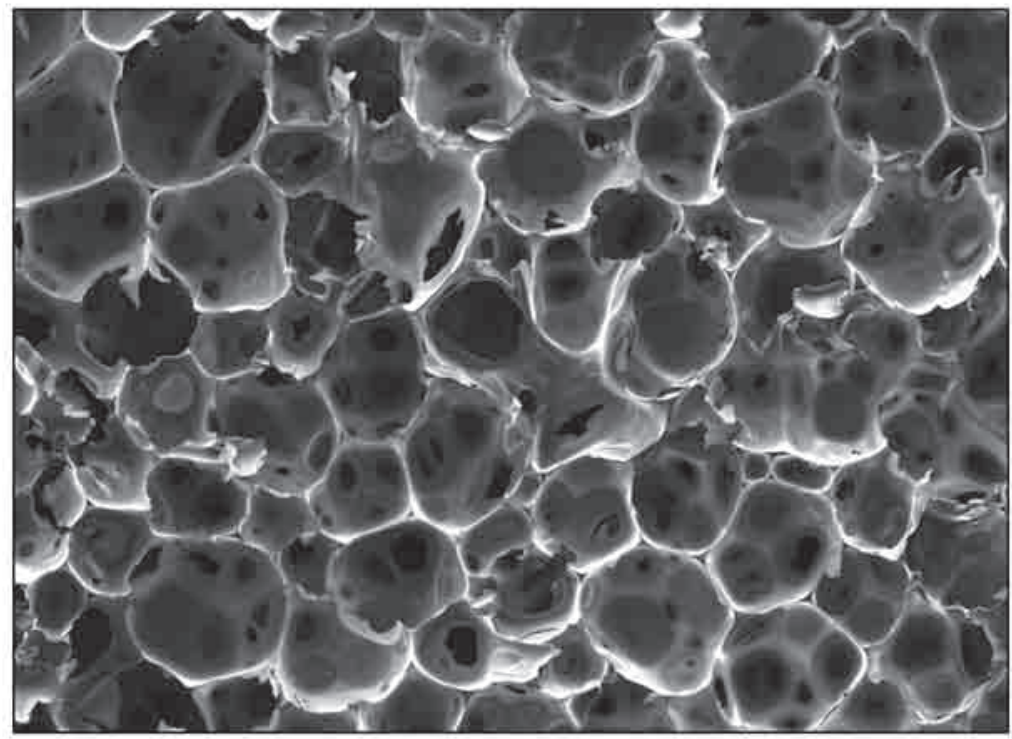

Figure 4. Scanning electron microscope image of detail of a finished tannin-based rigid foam at 30 magnification. Note the regularity of the foam cells and the distribution of the open ducts in the foam cells.

The use of insulating foams for construction cavities rests on two important parameters: (1) the capability of the foam to thermally insulate a structure and (b) the capability to withstand exterior humidity if the structure is exposed to the weather. The first property is essential for all applications both interior and exterior. Thus, for insertion in interior hollow-core doors, or hollow core wooden walls, the thermal conductivity of the foam itself, not that of the structure, has to be determined. The tests of thermal conductivity of different density tannin-based foams of different density are shown in Table 1 and Fig. 5. These test were done in both the growth direction of the foam as well as in the direction perpendicular to its growth. The results in Table 1 indicate first of all that such foams, composed at the 95\% level by weight of natural materials are indeed good insulating materials, the scale going from 0 for total insulation to 15 for total thermal conductivity. To compare their results with those typical of existing synthetic polymer foams, such as polyurethanes foams, the values for these at parity of density are of $0.04 \mathrm{~W} / \mathrm{m} \cdot \mathrm{K}$ in relation to $0.2 \mathrm{~W} / \mathrm{m} \cdot \mathrm{K}$ for the tannin foams. The synthetic polyuretane foams are then slightly better insulating, but not even a order of magnitude better. They have, however, the disadvantage that are totally oil-derived and that they burn with ease releasing toxic fumes, while tannin foams are 95\% natural materials and they do not burn at all (Meikleham et al.1994, Tondi et al. 2008). Fig. 5 shows also that thermal conductivity at these low density appears to be in practice independent of the foam density. Furthermore (Fig. 5), the thermal conductivity is slightly higher in the direction of foam growth than in the direction perpendicular to it, although such a difference is not very marked. Fig. 6 shows in colour the variation of the temperature of the foam sample within the hot plate and the cold plate. One can see that while the temperature of the hot plate is at $61^{\circ} \mathrm{C}$ only a very thin layer of the foam sample surface in contact with the hot plate is at approximately $35^{\circ} \mathrm{C}$, and this notwithstanding the sharp difference in temperature between the two metal plates that is used to induce heat flow. Again a clear indication of a good thermal insulation material. 
Table 1. Thermal conductivity values for tannin-based foams of different density in the direction of foam growth and perpendicular to the direction of foam growth

\begin{tabular}{c|c|c|c}
\hline Sample & Direction & Density $\left(\mathbf{g} / \mathbf{c m}^{3}\right)$ & Thermal Conductivity (W/m.K) \\
\hline DEE2 & Growth & 0,115 & 0,026 \\
\hline DEE3 & Growth & 0,091 & 0,03 \\
\hline DEE4 & Growth & 0,056 & 0,027 \\
\hline & & & 0,024 \\
\hline DEE2 & Perpend. & 0,115 & 0,026 \\
\hline DEE3 & Perpend. & 0,091 & 0,023 \\
\hline DEE4 & Perpend. & 0,056 & \\
\hline
\end{tabular}

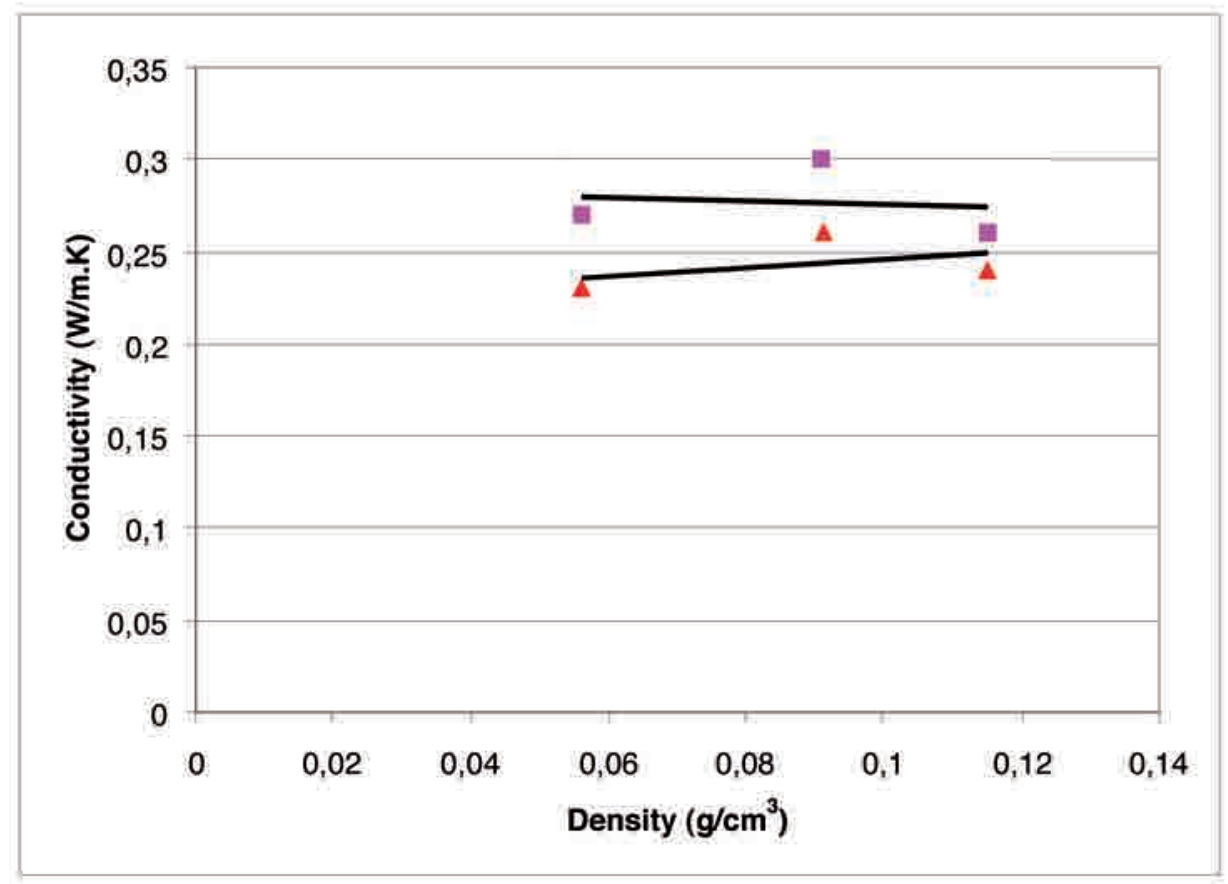

Figure 5. Thermal conductivity of tannin-based foams as a function of their density. 


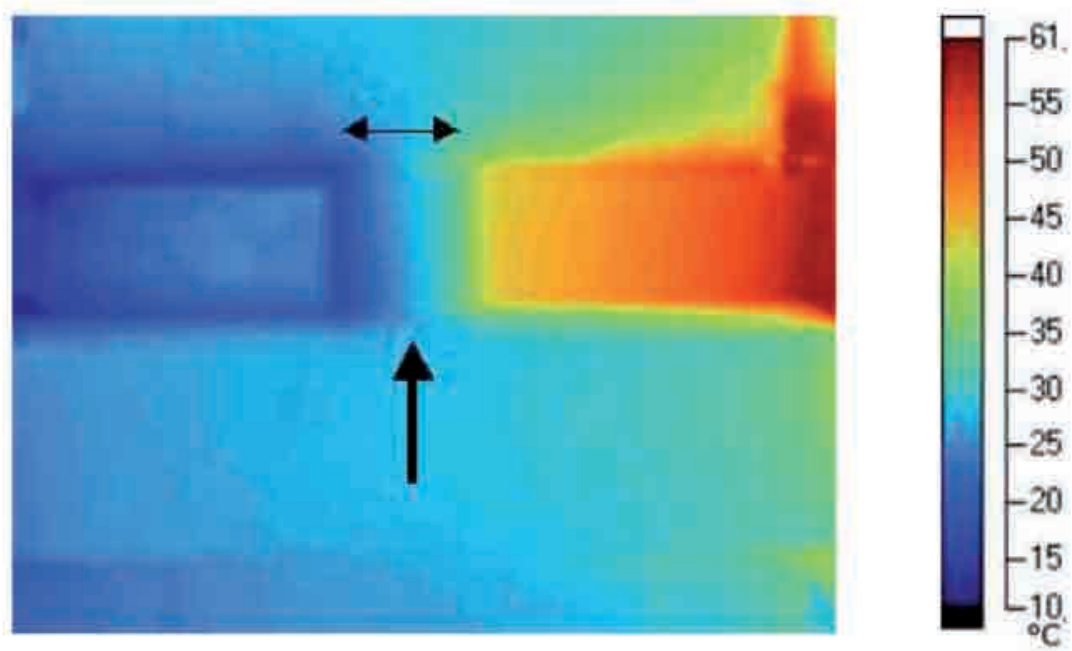

Figure 6. Thermogram indicating the temperature of the hot and cold metal plates. The arrow shows the position of the foam between the two metal plates. Note the low temperature of the foam surface in contact with the hot metal plate.

The second parameter of interest is the capability of the foam to withstand exterior humidity if the structure is exposed to the weather. This is of particular importance in the case of tannin-based foams because these are acid setting. Although not apt for structural applications, and by themselves alone being well capable to take any weather, as any phenolic resin can, their acidity may constitute a problem if the acid would attack the wood walls of the door in which the foam is encased. In Fig. 1 are shown the samples once the mix has been foamed within plywood boxes. The foam in excess, protruding from the boxes is then cut with a knife to give an even surface (Fig. 1) and a plywood lid nailed in place to close the box before exposure to the weather. Fig. 6 shows the conditions of a foam in a box open just before weather exposure (Fig. 2), and after the box has been forced open after 8 months exposure to the weather (Fig. 7) (exposure will still continue for two years). One can see that the foam, notwithstanding the damage due to the rough opening of the wooden box encasing it, is still in good conditions. Examination of the interior wood surface of the box with an optical microscope (Fig. 8) shows that no apparent bonding to the wood surface has occurred. It is well known, that the type of acid used in this foams becomes part of the network and is immobilized in the hardened foam, explaining the lack of damage of the wooden surface.

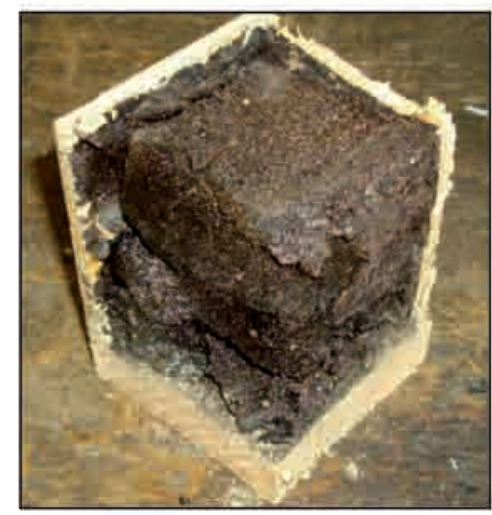

Figure 7. Appearance of plywood box as opened after 8 months exposure to wet winter weather. Note that the tannin-based foam is still in good condition. 


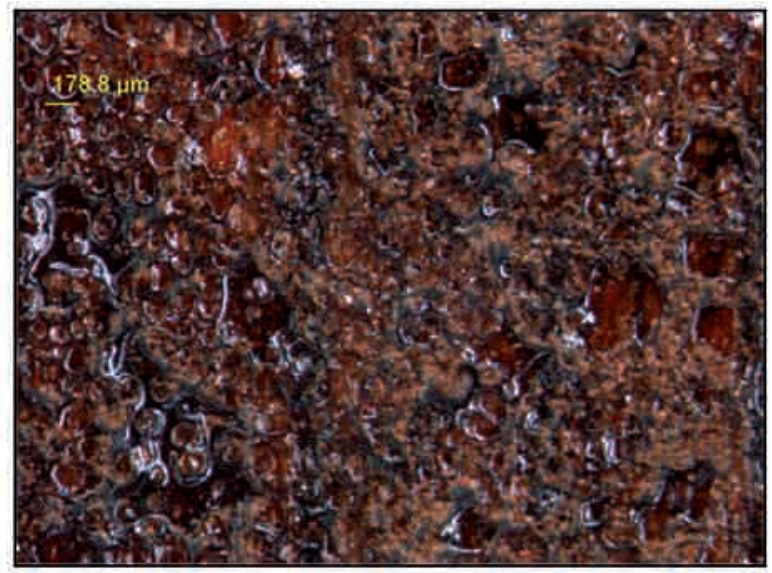

Figure 8. Optical microscope photograph of the interior wall of the plywood box in contact with the tannin-based foam after 8 months weather exposure. Photograph was taken after mechanically scraping the foam away from the wooden wall. Note excellent adhesion of residual foam to underlying plywood substrate.

Another important aspect for using tannin-based rigid foam as insulating material for domestic uses is their low level of formaldehyde emission. As no standard standard which evaluate the formaldehyde emission for these foams exists we adapted to foams the dessicator method for particleboard formaldehyde emission to try to obtain values which were comparable with the particleboard emission, (AS/ NZS 4266.16). The formaldehyde emission value obtained for a 8 months old tannin-based rigid foam was found to be $0.333 \mathrm{mg} / \mathrm{l}$ if the area of the geometrical surface of the cube is considered, thus as if the cube surface plane was completely smooth. This is already a good value if it is considered that the $F_{* * * *}^{*}$ Japanese emission standard (JIS A 5908) requires a value of $0.3 \mathrm{mg} / \mathrm{l}$ or lower. However good, this figure gives a wrong impression of the foam formaldehyde emission level. This is so because the typical surface of these foams is a reticulated porous structure having a real specific surface hundreds of times higher.

However the value obtain is three times smaller than what we obtain with a particleboard glued with a $U F \operatorname{resin}(F / U=1)$.

The active surface area of these foams has been calculated to be around $0,35 \mathrm{~m}^{2} / \mathrm{g}$ and that the whole weight of the sample tested is 16,2 $\mathrm{g}$. It means that the real contact area is 5,67 $\mathrm{m}^{2}$ instead of 453 $\mathrm{cm}^{2}$, hence 125 times higher (Tondi et al. 2008) than what used to calculate the value of $0.333 \mathrm{mg} / \mathrm{l}$ above. Thus the real emission based on actual surface of the foam is of only $0.0027 \mathrm{mg} / \mathrm{l}$. A very low value indeed. 


\section{CONCLUSIONS}

In conclusion, tannin-based rigid foams are good thermal insulating materials, 95\% of natural origin, that do not burn, and which can be used to fill hollow-core doors for interior and exterior applications. Their thermal insulation capacity is comparable to that of synthetic, oil-derived foams. They can be produced directly in the cavity of hollow-core wooden doors and other wooden cavities by injecting the liquid mixture and foaming the material in situ. They have the considerable advantage that because they do not burn they do not emit toxic gases on burning. Exposure to the weather of wooden hollow core structures filled with tannin-based foams confirm that the wood in contact with the foam is not affected by the acidity of the foam.

\section{REFERENCES}

Aguilar, R.; Ramırez, J.A.; Garrote , G.; Vazquez, M. 2002. Kinetic study of the acid hydrolysis of sugar cane bagasse. J. Food Eng. 55: 309-318.

Australian/New Zealand Standard 2004. AS/NZS 4266.16. Reconstituted wood-based panels -Methods of test. Method 16: Formaldehyde emission - Dessicator method,

Japanese Standards Association. 1994. JIS A 5908. Particleboards. Japanese Standards Assoc., Tokyo, Japan.

Nagaraja, B.M.; Siva Kumar, V.; Shasikala, V.; Padmasri, A.H.; Sreedhar, B.; David Raju, B.; Rama Rao, K.S. 2003. A highly efficient $\mathrm{Cu} / \mathrm{MgO}$ catalyst for vapour phase hydrogenation of furfural to furfuryl alcohol. Catal. Comm. 4: 287-293.

Meikleham, N.E.; Pizzi, A. 1994. Acid-and Alkali-Catalyzed Tannin-Based Rigid Foams. J. Appl. Polym. Sci. 53: 1547-1556.

Pizzi, A. 1994. Advanced Wood Adhesives Technology. Dekker, New York.

Pizzi, A.; Tondi, G.; Pasch, H.; Celzard, A. 2008. Maldi-ToF Structure determination of complex thermoset network - Polyflavonoid tannin-furanic rigid foams. J. Appl. Polym.Sci. in press (2008).

Tondi, G.; Fierro, V.; Pizzi, A.; Celzard, A. 2008. New tannin-based carbon foams. Carbon, in press 2008

Tondi, G.; Pizzi, A. 2008. Tannin-based rigid foams: Characterization and modification. Ind. Crops and Prod. In press : doi:10.1016/j.indcrop.2008.07.003

Tondi, G.; Pizzi, A.; Pasch, H. Celzard, A. 2008. Structure degradation, conservation and rearrangement in the carbonization of polyflavonoid tannin/furanic rigid foams - a MALDI - TOF investigation. Polymer Degrad. \& Stabil. 93(5): 968-975

Touloukian, Y.S.; Powell, R.W.; He, C.Y.; Klemens, P.G. 1970. Thermal conductivity. In Thermophysical Properties of Matte 2:l-39. IFl/Plenum, New York-Washington. 
